\section{Bluttransfusion bei Herzoperation: Es geht auch auf restriktive Weise}

\author{
Herzoperationen verursachen Blutverluste. Die Zahl der Bluttransfusionen \\ lässt sich in solchen Fällen durch eine restriktive Indikationsstellung erheblich \\ verringern.
}

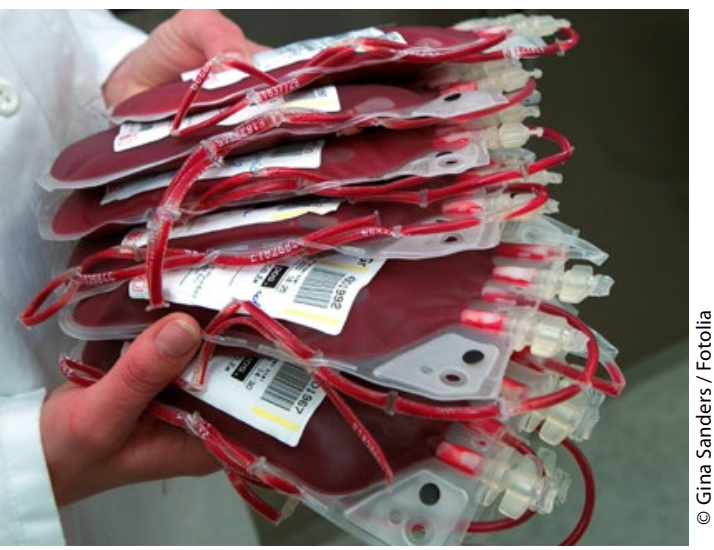

Es müssen nicht immer so viele Bluttransfusionen sein. Dem Patienten schadet es nicht, wenn er bei einem $\mathrm{Hb}$ von $8 \mathrm{~g} / \mathrm{dl}$ keine bekommt.

$B^{\ln }$ utverluste lassen sich bei Herzoperationen kaum vermeiden. Bluttransfusionen können eine Anämie verhindern, die bekanntlich mit einer erhöhten Mortalität assoziiert ist. Andererseits gehen Transfusionen von Fremdblut aber auch mit Risiken einher. Soll in dieser Situation also eher nach restriktiven oder liberalen Kriterien über eine Transfusion entschieden werden? Nach derzeitiger Studienlage gibt es darauf keine definitive Antwort.

Die TRICS-III-Studie sollte eine Entscheidungshilfe liefern. 5.243 für eine Herzoperation vorgesehene Patienten mit einem mittleren bis hohen Operationsrisiko sind auf beide Transfusionsstrategien randomisiert worden. In der Gruppe mit restriktiver Indikation erhielten die Patienten Bluttransfusionen, sobald das Hämoglobin (Hb) im OP oder auf der Intensiv- und Normalstation auf unter 7,5 g/dl gefallen war. Bei der liberalen Strategie erfolgte eine Transfusion bereits dann, wenn der Hb-Wert auf unter 9,5 g/dl (unter 8,5 mg/dl auf der Normalstation) gesunken war.

Je nach Strategie war der Anteil der Patienten, die eine oder mehrere Blut- transfusionen erhalten hatten, in beiden Gruppen erwartungsgemäß unterschiedlich: Bei restriktiver Indikation war er mit 52,3\% auch nicht gerade niedrig, bei liberaler Indikation mit 72,3\% aber deutlich höher.

Auf den klinischen Verlauf wirkte sich dieser Unterschied nicht aus. Maßgeblich für dessen Beurteilung war die Rate der Ereignisse Tod, Myokardinfarkt, Dialyse wegen neu aufgetretenem Nierenversagen und neue fokale neurologische Defizite (primärer kombinierter Endpunkt) zum Zeitpunkt der Klinikentlassung bzw. nach vier Wochen. Mit $11,4 \%$ (restriktive Indikation) und $12,5 \%$ (liberale Indikation) unterschieden sich die Ereignisraten nur wenig.

Mit Ausnahme von Herzinfarkten fielen die Ergebnisse bei allen anderen Komponenten des primären Endpunkts zumindest numerisch zugunsten der restriktiven Vorgehensweise aus. Etwas überraschend schien diese Strategie speziell bei älteren Patienten besonders vorteilhaft gewesen $\mathrm{zu}$ sein.

Die statistischen Kriterien für „NichtUnterlegenheit" der restriktiven Strategie wurden damit klar erfüllt. Auch bzgl. der sekundären Endpunkte wie Dauer des Klinikaufenthalts, akute Nierenschädigung oder Infektionen gab es keine nennenswerten Unterschiede.

Demnach kann bei Herzoperationen auf viele Bluttransfusionen ohne Nachteil für die Patienten verzichtet werden. Das wirkt sich auch auf die Behandlungskosten vorteilhaft aus: Studienleiter Mazer taxierte die allein im Rahmen der Studie mit der restriktiven Strategie erzielte Kosteneinsparung auf rund 3 Millionen Dollar. Peter Overbeck

Quelle: „Late-breaking Science 1“ (LBS 01): TRICS III - An International Multicenter Randomized Trial of Transfusion Triggers in Cardiac Surgery, Kongress der American Heart Association (AHA) 2017, 11.-15.11.2017 in Anaheim; Mazer CD et al. N Engl J Med. 2017, doi: 10.1056/NEJMoa1711818

\section{Welche Ernährung schützt das Herz am besten?}

Aufgrund der Flut an Ernährungsstudien wird sich bei einigen Patienten vermutlich schon Resignation breit gemacht haben. Was denn nun, "Low Carb", vegetarisch, Schokolade, Rotwein? Das Sammelsurium an vermeintlicher Evidenz zur bestmöglichen gesunden Ernährungsweise wird nun durch eine weitere Beobachtungsstudie erweitert.

Diesmal geht es darum, welche Ernährung das Herz bei bereits kardiovaskulär erkrankten Patienten am besten schützt. Fünf für US-Amerikaner offenbar typische Ernährungsgewohnheiten traten in den Wettstreit:

_Der sog. "convenience“-Style: typisches mexikanisches und chinesisches Essen, also sowohl Fleisch als auch Bohnen.

_-Pflanzliche Ernährung: mit viel Obst, Gemüse, Säften, Getreide, Fisch und Geflügel.

_Die „süße“ Ernährungsgewohnheit, mit Vorliebe für Schokolade, Desserts und süßem Frühstück, daneben gibt's Brot und generell viel Fett.

— "Southern"-Style: Hier steht ebenfalls viel Fett und Frittiertes auf dem Speiseplan, ebenso wie verarbeitetes und unverarbeitetes Fleisch, fettreiche Milch und Eier.

_ Die als "Salat/Alkohol“" klassifizierte Ernährungsweise, bei man sich zum Salat, schmackhaft gemacht durch ein Salatdressing, öfter mal ein Glas Wein, Bier oder sonstige alkoholische Getränke genehmigt viel Nüsse und Kaffee konsumiert.

Die Einteilung basiert auf einer Befragung von 15.569 Teilnehmern der REGARDS-Studie, die an einer

Herzschwäche oder KHK erkrankt waren.

Das Ergebnis nach über 3.000 Tagen Follow-up favorisiert die vegetarische Ernährungsweise. Jene Patienten, die diese am meisten befolgten, hatten ein um $28 \%$ geringeres Risiko in dieser Zeit wegen einer Herzinsuffizienz in eine Klinik eingewiesen zu werden, als jene, die die anderen Ernährungsvorlieben teilten.

Die Studienautorin empfiehlt fünf bis sieben Portionen Früchte oder Gemüse am Tag zu essen, Fertiggerichte und Frittiertes zu meiden, und stattdessen mageres Fleisch wie Geflügel oder Fisch zu essen.

Man darf gespannt sein auf weitere retrospektive Studien, bei denen sich womöglich neue Zusammenhänge auftun werden. Veronika Schlimpert 